\title{
Ranking Popular Items By Naive Bayes Algorithm
}

\author{
Shiramshetty Gouthami ${ }^{1}$, Golamari.Jose Mary ${ }^{2}$ and Pulluri Srinivas Rao ${ }^{3}$ \\ ${ }^{1}$ Department of Computer Science and Engineering, JNTUH, Jayamukhi Institute of \\ Technological Sciences,Narsampet, Warangal, Andhrapradesh-506332, India \\ gouthami.shiramshetty@gmail.com \\ ${ }^{2}$ Department of Computer Science Engineering, JNTUH, Jayamukhi Institute \\ ofTechnological Sciences,Narsampet, Warangal, Andhrapradesh-506332, India \\ sreyas.2007@yahoo.com \\ ${ }^{3}$ Department of Computer Science Engineering, JNTUH, Jayamukhi Institute of \\ Technological Sciences,Narsampet, Warangal, Andhrapradesh-506332, India \\ srimaraodyahoo.co.in
}

\begin{abstract}
The problem of ranking popular items is getting increasing interest from a number of research areas. Several algorithms have been proposed for this task. The described problem of ranking and suggesting items arises in diverse applications include interactive computational system for helping people to leverage social information; in technical these systems are called social navigation systems. These social navigation systems help each individual in their performance and decision making over selecting the items. Based on the each individual response the ranking and suggesting of popular items were done. The individual feedback might be obtained by displaying a set of suggested items, where the selection of items is based on the preference of the individual. The aim is to suggest popular items by rapidly studying the true popularity ranking of items. The difficulty in suggesting the true popular items to the users can give emphasis to reputation for some items but may mutilate the resulting item ranking for other items. So the problem of ranking and suggesting items affected many applications including suggestions and search query suggestions for social tagging systems. In this paper we propose Naïve Bayes algorithm for ranking and suggesting popular items.
\end{abstract}

\section{Keywords}

Label ranking, suggesting, computational systems, collaborative filtering, preferential attachment, mutilate, true popular item sets, tagging systems, suggested itemsn and ranking rules.

\section{INTRODUCTION}

\section{About Naive Bayes}

Our work is completely based on the Bayes theory. We want to propose Naive Bayes algorithm that is based on conditional probabilities. Ranking uses Bayes theory concepts.A Bayes theorem is a mathematical formula that calculates probability by including the frequency of values and combinations of values in the chronological data. Bayes theorem finds the probability of an event occurring in the probability of another event that has already occurred. If $B$ represents the dependent event and A represents the prior event, then the Bayes' theorem can be stated as follows. 
Bayes' Theorem, published posthumously in the eighteenth century by Reverend Thomas Bayes, says that you can use conditional probability to make predictions in reverse! It is very powerful.

For Naïve Bayes, supervised binning is performed by automatic data preparation. Decision trees are used by supervised binning to create the optimal bin boundaries. Both categorical and numerical attributes are binned.

The missing values in Naive Bayes are handled naturally as missing at random. The Naïve Bayes algorithm replaces sparse numerical data with zeros and sparse categorical data with zero vectors. The missing values in nested columns are interpreted as sparse and the missing values in columns with simple data types are interpreted as missing at random.

For example, if you want to manage your own data preparation, remember that Naive Bayes usually requires supervised binning. Naive Bayes totally relies on counting techniques for calculating probabilities. The columns should be binned to reduce the cardinality as suitable. The Numerical data can be binned into ranges of values like low, medium, and high. The categorical data can be binned into meta-classes like regions instead of cities. Equal-width binning is not suggested, since outliers will cause most of the data to concentrate in a few bins, sometimes a single bin. As a result, the discriminating power of the algorithms will be significantly reduced

We suppose the problem of learning the popularity of items that is considered to be a priori unidentified but have to be learned from the observed user's selection of items. Especially, we consider systems in which each user is presented with a list of items called suggested items and the user selects a set of favored items that can contain either suggested items or any other items preferred by the user. The inventory of suggested items would naturally contain only a small subset of popular items. The goal of any inventory system is to efficiently learn the popularity of items and suggest popular items to users. The items are suggested to users to make easy tasks such as browsing or tagging of the content.

Items could be search query keywords, files and any items selected by users from short lists of popular items. A precise application is that of tagging of the content where items are tags applied by users to content such as books details for their later retrieval or personal information management. The basic idea of social tagging is that the user can select any set of tags for an information object according to her/his preference. In the majority existing social tagging applications, users are offered with tag suggestions that are made based on the history of tag selections. The process of learning of item popularity is complicated by the suggesting of items to users. In reality, we expect that users would be inclined to select suggested items more frequently. There are various reasons for this could happen. For example, "least effort " where users select suggested items, as it is easier than thinking of alternatives. Wherever humans may be likely to conform to choices of other users that are reflected in the suggestion set presenting a few popular items. Actually, we find indications that such popularity prejudice may happen. If the process of suggesting popular items seems to be difficult due to potential popularity disorder, why can't we make suggestions in the first place? There are several reasons for this. For example, sometimes suggestions may help to remember what candidate items are. A fasten to avoid popularity slant would be to suggest all candidate items and not restrict to a small list of few popular items. But this is often impractical for the reasons such as limited user interface space, user's capability to process smaller sets easier, and the triviality of less popular items. Consequently, the number of suggestion items in the item set is limited to a small number.

In this paper, our goal is to propose Naïve Bayes algorithm for popular items to users in a way that enables learning of the users' true preference over items. The factual first choice refers to the preference over items that would be pragmatic from the user's selections over items without revelation to any suggestions. A simple scheme for ranking and suggesting popular items (that 
appears in common use in practice) presents a fixed number of the most popular items as observed from the past item selections. We illustrate an analysis that suggests a simple system with a security device to lock down to a set of items that are not the truest popular items if the popularity prejudice is sufficiently large, and may vague learning the true predilection over items.

In this paper, we propose Naïve Bayes algorithm designed to avoid such fortifications and offer strict performance analysis of the ranking boundary points and popularity of the suggested items.

The probability in Naive Bayes algorithm is calculated by dividing the percentage of pair-wise occurrences by the percentage of singleton occurrences. If the percentages that are calculated are very small for a known predictor, they probably will not contribute to the effectiveness of the model. The occurrences below a certain threshold value can usually be disregarded. The Naive Bayes algorithm provides a fast, highly scalable model. The algorithm scales linearly with the number of predictors and rows. The put up process for Naive Bayes is parallelized. It means that scoring can be parallelized irrespective of the algorithm.

Both binary and multiclass classification problems use Naïve Bayes Algorithm.

According to the definition of conditional probability: [1] $\mathrm{P}(\mathrm{B} \mid \mathrm{A})=\mathrm{P}(\mathrm{A}$ and $\mathrm{B}) / \mathrm{P}(\mathrm{A})$ Bayes' Theorem is used to solve for the inverse conditional probability, $\mathrm{P}(\mathrm{AlB})$. By definition, [2] $\mathrm{P}(\mathrm{A} \mid \mathrm{B})=\mathrm{P}(\mathrm{A}$ and $\mathrm{B}) / \mathrm{P}(\mathrm{B})$ Solving [1] for $\mathrm{P}(\mathrm{A}$ and $\mathrm{B})$ and substituting into[2] gives Bayes'Theorem:

$\mathrm{P}(\mathrm{A} \mid \mathrm{B})=[\mathrm{P}(\mathrm{B} \mid \mathrm{A})][\mathrm{P}(\mathrm{A})] / \mathrm{P}(\mathrm{B})$

Using Bayes'Theorem,

$\mathrm{P}(\mathrm{A} \mid \mathrm{B})=[\mathrm{P}(\mathrm{B} \mid \mathrm{A})][\mathrm{P}(\mathrm{A})] / \mathrm{P}(\mathrm{B})$

Example 1 for Bayes theorem:-

event

description

probability

A

Drawer A has two gold coins

B

Person chooses a gold coin out of the four coins

0.75

$\mathrm{B} \mid \mathrm{A}$ Conditional probability of choosing a gold coin from $\mathrm{A}$ if it has two gold coins

Using Bayes' Theorem,we have

$\mathrm{P}(\mathrm{A} \mid \mathrm{B})=[\mathrm{P}(\mathrm{B} \mid \mathrm{A})][\mathrm{P}(\mathrm{A})] / \mathrm{P}(\mathrm{B})=[1.0][0.5] /[0.75]=2 / 3$

Example 2 for Bayes theorem:-

event

A

B Someone has microscopic hematuria

BIA Conditional probability of having hematuria given kidney cancer 1.0

\section{probability}

0.000002

0.10 
International Journal of Computer Science \& Information Technology (IJCSIT) Vol 4, No 1, Feb 2012

Using Bayes'Theorem, we have

$\mathrm{P}(\mathrm{A} \mid \mathrm{B})=[\mathrm{P}(\mathrm{B} \mid \mathrm{A})][\mathrm{P}(\mathrm{A})] / \mathrm{P}(\mathrm{B})=[1.0][0.000002] /[0.1]=\quad=\quad 00002$

That is, you still have a very low probability of kidney cancer.

$\operatorname{Prob}(\mathrm{B}$ given $\mathrm{A})=\operatorname{Prob}(\mathrm{A}$ and $\mathrm{B}) / \operatorname{Prob}(\mathrm{A})$

\section{Previous work}

We consider the problem of learning the popularity of items based on user feedback. In particular, we consider a system where each user is presented with a set of items ("suggested items"), and the user can then select her set of preferred items either choosing items from the suggestion set, or by choosing any other item. Here we are interested in the situation where the set of suggested items contains only a small subset of all possible items. The goal is to design an algorithm for ranking and suggesting items such that system can quickly learn the true popularity of individual items. The problem of how to rank and suggest items to users arises in several applications including social tagging appli- cations, browsing components of some web application (e.g. del.icio.us' "tags to watch" list containing a list of tags and "popular" page containing a list of urls), and search en-gines. In the context of social tagging applications, items to be ranked and suggested correspond to the tags (key- words) that users attach to information objects such as pho- tos (e.g. Flickr), videos (e.g. YouTube), or web pages to users a set of suggested tags based on the tagging history of a given itemThe difficulty that arises in the above problem is that sug- gesting items to users can reinforce the popularity of items, and possibly distort the resulting ranking. This will hap- pen if users are likely to choose items from the suggestion set. This behavior can be seen as a "bandwagon problem", i.e. user's choice is swayed toward items from the sugges- tion sets due to a tendency to conform to the choice of users that already made their selections, or just because it takes less (cognitive) effort to choose from the presented set of items. That the bandwagon problem indeed arises in so-cial tagging scenarios is suggested by the work of Sen et al [20] In our analysis, we show that "bandwagoning" can indeed be a problem by considering an algorithm for suggesting items (that appears to be in com- mon use in practise) which presents to users a fixed number of the top most popular items as observed from the past user selections. We show that if the bandwagoning is sufficiently large, then this scheme (TOP) can lock down to a set of items that does not correspond to the set of the most popular items. Motivated by this observation, we then consider several algorithms for ranking and suggesting of popular items with the goal to (a) learn the true popularity ranking of items, (b) suggest popular items, and (c) identify the popular items quickly. In particular, we propose and study the performance of three randomized update rules for making item suggestions based on the history of the item selections. Two of our proposed algorithms are lightweight in their computational and storage requirements.Our proposed schemes enable dis- playing a larger set of popular items over time than can be accommodated into a single suggestion set, which may be of interest in application scenarios where the size of the sug-gestion size is limited and is desired to display a larger set of popular items than can fit into a single suggestion set.

User Model: Part of our analysis is based on a user model that is summarized as follows. Each user selects an item according to her true pref- erence over items either from the entire set of items or from the suggestion set of items. We call the 
probability with which the latter case happens, the "imitation probability" and evaluate the robustness in learning the true popularity of items with respect to this parameter of the suggestion set size and the true popularity ranks of items. This result enables us to estimate the threshold im- itation probability for given true popularity ranks of items. We have done this using the inferred popularity rank scores of the tags from our dataset where we find that the median imitation probability over the bookmarks in the dataset is around 0.1 for the suggestion set sizes ranging from 1 to 10 tags. This result suggests that in real-world scenarios using the simple scheme TOP may result in failing to learn the true popularity of items already at a small level of imitation. We next discuss the three randomized algorithms that we consider in this paper. First, we consider a randomized algorithm(PROP) that suggests to each user a random set of items $S$ sampled with the probability proportional to the sum of the current item popularity rank scores.

We call this algorithm PROP (frequency pro-portional sampling). Sampling the suggestion set of items proportional to the sum of the item popularity rank scores appears a natural randomisation strategy that one would consider to avoid the popularity ranking skew, letting each item appearing recurrently in the suggestion set. We show, however, that this is guaranteed only if the imitation prob- ability is smaller than a threshold and fully specify this threshold. Another issue with PROP is that the frequency proportional sampling can be computationally demanding. In the sequel, we present two algorithms that are computa- tionally lightweight.We second consider a randomized, recursive update rule for the suggestion set of items described as follows. When- ever a user selects an item that is in the suggestion set pre- sented to this user, nothing happens. Otherwise, the item replaces a randomly evicted item from the suggestion set.We call this algorithm M2S, alluding to the apparent "move to set" feature of the algorithm. The reader may note that the algorithm biases to showing recently used items; for the special case of the suggestion set size equal to 1 item, the algorithm corresponds to showing the last used item. It is worth noting that the M2S does not require using the coun- ters for the number of per-item selections. We will see that this rule tends to sampling the suggestion set of items pro- portional to the product of the item true popularity rank scores. We show that M2S combined with ranking the pop-ularity of items with respect to the number of per-item se- lections guarantees to learn the true popularity ranking, for any imitation probability $<1$. This is a very interesting roperty suggesting robustness of the M2S update rule to the users' imitation. Note that under algorithm M2S, any item selected by a user that was not suggested to this user, replaces an item in the suggestion set. Hence, any item that is recurrently selected by users (no matter how small the fre- quency of selection is) appears recurrently in the suggestion set. We next consider an algorithm that tends to displaying only sufficiently popular items, which may be preferred in some applications.

We third consider the randomized algorithm FM2S ("fre-quency move-to-set") described as follows.At a high level,FM2S replaces an item in the suggestion set with a new item only if this new item is (likely to be) more popular than at least one item already in the suggestion set.The algorithm can be seen as TOP but with the rank scores redefined to the number of per-item selections updated only when an item is selected and was not suggested. This is done to mitigate the positive reinforcement of the item popularity due to exposure in the suggestion set. We show that FM2S tends to displaying a subset of sufficiently popular items with respect to the true popularity and fully determine this set in terms of the suggestion set size and the true popularity rank scores of items. The ranking of these items can be inferred from their frequency of appearance in the 
suggestion set. This algorithm proposal can be seen as a relaxation of TOP that avoids locking down to suggesting a set of items that are not the true most popular. It enables displaying a larger set of true most popular items than it can be accommodated into a single suggestion set, which may be of interest in practise. As a final point, we present numerical results obtained by evaluating our analytical results using the popularity rank scores of tags for bookmarks derived from a month-long crawl of the social bookmarking application delectable.

We consider user selection over $C>1$ items and denote this set as $C:=\{1,2, \ldots$, C $\}$. Let us consider $\mathbf{r}=(\mathbf{r} 1, \mathrm{r} 2, \ldots, \mathrm{rC})$ be the user's true preference over the set of items $\mathrm{C}$, and call $\mathbf{r}$ the true popularity rank scores. For an item $\mathrm{i}$, we interpret $\mathbf{r} \mathbf{i}$ as the portion of users that would select item $\mathbf{i}$ if suggestions were not made. We assume that the true popularity rank scores $\mathbf{r}$ are such that (a) $\mathbf{r i}_{\mathbf{i}}$ is strictly pos- itive for each item $i$, (b) items are enumerated such that $\mathbf{r} 1 \geq \mathbf{r} 2 \geq \cdots \geq$ $\mathbf{r C}$, and (c) $\mathbf{r}$ is normalized such that it is a probability distribution, i.e. $\mathbf{r} 1+\mathbf{r} 2+$ $\cdots+\mathrm{rC}=1$. An algorithm is specified by (a) ranking rule: the rule that specifies how to update the ranking scores of items, denoted as $\rho=(\rho 1, \ldots, \rho \mathrm{C})$ and (b) suggestion rule: this rule specifies that, what subset of items to suggest to a particular user. We suppose that the size of the suggestion set is fixed to $\mathrm{s}$, a positive integer that is a system configuration parameter.

The design objective to learn the true popularity ranking if items means that the ranking order induced by $\rho(t)$ is the same as that induced by the true popularity ranking scores $\mathrm{r}$, as the number of users $\mathrm{t}$ tends to be large. In other words, we want that for any two items $\mathbf{i}$ and $\mathbf{j}, \mathbf{r i} \geq \mathbf{r j}$ implies $\rho \mathbf{i}(t) \geq \rho \mathbf{j}(t)$, for sufficiently large $t$. The design objectives are also to suggest true popular items and to identify quickly the true popular items (ideally, we would like that the ranking order induced by $\rho(t)$ conforms to that induced by $r$, after a small number if item selections). Let $\mathrm{s}$ be the size of the set $\mathrm{S}$. Let $\mathrm{v}$ be the smallest positive integer $\mathrm{s} \leq \mathrm{v}<\mathrm{C}$ such that $\mathrm{rs}_{\mathrm{s}}=$. $\cdots=\mathrm{r}_{\mathrm{v}}<\mathrm{r}_{\mathrm{v}+1}$ (if $\mathrm{r}(\mathrm{s})=\cdots=\mathrm{rC}$, then set $\mathrm{v}=\mathrm{C}$ ). If item $\mathrm{s}$ is strictly more popular than item $\mathrm{s}+1$, then $\mathrm{v}=\mathrm{s}$.

User's choice model: the user selects an item over a set of 8 items and is suggested the set of items $S=\{2,4,5,7\}$.With probability $1-\mathrm{pS}$, the user selects an item by sampling from the distribution $r$ over the entire set of items, else, the same but confined to the items in the set $\mathrm{S}$.is thus by a fixed multiplicative factor greater than one We then have the imitation probability pS $:=[(\alpha-1) \mathrm{rS}] /[1+(\alpha-1) \mathrm{rS}]$ where $\mathrm{rS}:={ }_{\mathbf{j} \in S}^{\mathbf{r} \mathbf{j}}$ .Note that given the suggestion set of items, the user's item selection is stochastically independent of the past item selections. This may not hold if we consider items selected.ere $1 \mathrm{~A}=1$ if $\mathrm{A}$ holds, and $1 \mathrm{~A}=0$ else. In other words, $\operatorname{prec}(\mathrm{S})$ is the fraction of most popular items $1,2, \ldots, \mathrm{v}$ that are in the set $\mathrm{S}$. This is a standard information-retrieval measure of precision $[18,21]$ defining the set of relevant items as the most popular items $1,2, \ldots, \mathrm{v}$.

2.1 User's choice model :- We introduce a user's choice model defined as follows. Suppose a user is presented a set $S$ of suggested items. The user selects an item from the entire set of items by sampling using the true item popularity distribution $r$, with probabil- ity $1-\mathrm{pS}$.Otherwise, the user does the same but confines her choice to items in the suggestion set $\mathrm{S}$. In other words,

\subsection{A Naïve Algorithm}

We first introduce the simple algorithm TOP which consists ranking and a suggestion 
rule as defined below.

\section{TOP (Top popular)}

Init: $\mathrm{V}_{\mathbf{i}}=0$ for each item $\mathbf{i}$

At the t-th user selection:

If item i selected:

$\mathrm{V}_{\mathbf{i}} \leftarrow \mathrm{V}_{\mathbf{i}}+1$

$\mathrm{S} \leftarrow$ a set of $\mathrm{s}$ items with largest $\mathrm{V}$ counts

Fig:1 TOP Algorithm.

The ranking rule is to set the rank score of an item equal to the number of selections of this item in the past. For this algorithms and the algorithms introduced later, we initialize $\mathrm{Vi}=0$ for each item i. The implicit assumption is that we assume no prior information about the popularity of items, and hence, initially assume that all items are equally popular.

The suggestion rule sets the suggestion set to a set

$=(1-\mathrm{pS}) \underset{\mathbf{j}}{\mathbf{r} \mathrm{i}}+\mathrm{pS} \quad-2$

the top s most popular items with respect to the current popularity rank scores. We admitted this simple and intuitive model in order to facilitate analysis under a model of user's choice that bi- ases to items in the suggestion set. The user's choice model accommodates two special cases:

Case 1: Let us consider a dichotomous user population where a fraction $1-\mathrm{p}$ of users sample an item from the distribution $r$ over the entire set of items and the remaining fraction of users, $p$, imitate by sampling an item from their preference distribution $r$ confined to the presented suggestion set. We then have that Eq. (2) holds with $\mathrm{pS} \equiv \mathrm{p}$.

Case 2: Suppose that suggesting an item boosts its proba-bility of selection in the following particular way. Each user selects an item i with probability proportional to $\alpha \mathbf{i}$ where $\alpha>1$ if item $\mathbf{i}$ is suggested and $\alpha=1$ if item $i$ is suggested. The boost of items presented in the suggestion set

We will later identify cases when this simple algorithm can get locked down to a ranking $\rho$ that induces different ranking than that induced by the true popularity ranking $\mathrm{r}$, and thus, may fail to learn the true popularity of items. To overcome this problem, we consider in the following alterna- tive ranking and suggestion rules.

\subsection{Ranking Rules}

In this subsection, we define two ranking rules called rank rule 1 and rank rule 2 .

Rank Rule 1. A simple ranking rule is the one that we already encountered in the algorithm TOP, where the rank

In practise, one may use prior information about item pop- ularity. For example, in social bookmarking applications, in- formation from the keywords meta-data and content of web pages can be used to bias the initial tag popularities.

score for an item $\mathbf{i}$ is incremented by 1 whenever a user selects this item.

Init: $\mathrm{V}_{\mathbf{i}}=0$ for each item $\mathbf{i}$ 
At the t-th user selection:

We will see that this ranking may fail to discover the rank- ing order of the true popularity when combined with a sug- gestion rule that reinforces candidates that were selected early on, as it is the case under the algorithm TOP.

Rank Rule 2. We have noted that the rank rule 1 might fail to determine the ranking order of the true popularity of items. To come out this problem, we may change the ranking rules(scores) in the following in the following way.

Now, the rank scores $\rho$ are updated only for an item that is selected by a user and was not suggested to this user. The ranking score $\rho \mathbf{i}$ for an item $\mathbf{i}$ can be interpreted as the rate of user selections of item $i$ over users that were not suggested item $i$. We have the following result:

Lemma 1. Let us consider any suggestion rule combined with the rank rule 2 under the only assumption that each item exits the suggestion set infinitely often. Then, under the user's choice model which is rational to the sum of the current rank scores of items. The algorithm is described in more detail below:

\section{PROP (Frequency proportional)}

At the t-th user selection:

Sample a set $\mathrm{S}$ of $\mathrm{s}$ items with probability

Fig 2: PROP Algorithm.

We will later show analysis suggesting that this suggestion rule combined with rank rule 1 is more robust to imitation than TOP, but there still may exist cases when it fails to learn the true popularity of items. Note also that the algorithm is computationally demanding when the number of items $\mathrm{C}$ and suggestion set size $\mathrm{s}$ are non small; it requires sampling on a set of $\mathrm{C}$ elements. Our next algorithm is computationally very simple.

\section{M2S (Move-to-set)}

At the t-th user selection with item i selected:

If item $\mathrm{i}$ not in the suggestion set $\mathrm{S}$

Remove a random item from $\mathrm{S}$ Add $\mathrm{i}$ to $\mathrm{S}$

Fig:3 M2S Algorithm.

The algorithm M2S is a randomized iterative update rule of the suggestion set of true popular items. The given suggestion set is updated only when a user selects an item that is not in the suggestion set presented to the user. Make a note of that for the suggestion set size of one item,M2S suggests the last used item, a recommendation rule used by many user interface designs. For the suggestion set size greater than one item, M2S is different from suggesting the last $\mathrm{s}$ distinct used items due to the random eviction of items from the suggestion set, but note that the rule does bias to presenting recently used items. We will present how exactly this update rule tends to bias the sampling of the suggestion set with respect to true popularity rank scores of items. Note also that M2S relates to the self-organized sorting of items known as move-to-front heuristic $. \operatorname{Lim} \mathbf{t} \rightarrow+\infty(\mathrm{t})=$ r.It follows from the description 
of the suggestion rule M2S that any item would recurrently appear in the suggestion.The result tells us that under the user's choice model, the rank rule 2 combined with a suggestion rule from broad set guarantees to learn the true popularity ranking of items, where this set comprises all suggestion rules for which each item exists the set with a probability that is lower bounded by a positive (but possibly very small) constant. The rank rule 2 might have a slow rate of convergence as the rank scores are updated only over a subsequence of item selections when they were not suggested. For this reason, we will focus in the following on rank rule 1.

\subsection{SuggestioRules}

We introduce three different suggestion rules: (a) Fre- quency Proportional (PROP), (b) Move-to-Set (M2S), and (c) Frequency Move-to-Set (FM2S).PROP is a randomized algorithm that for each user presents a suggestion set of items, sampled with probability propor- tional to the sum of the current rank scores of items. Having rank rule 1 conforming to the true popularity rank-ing means that the true popularity ranking of items can be inferred from the resulting item selections made by users. This may be of interest in practise, as one does not need additional information besides a sample of item selections to infer the popularity ranking of items.t, provided only that it is recurrently selected by users with some positive probability (no matter how small). We call this new algorithm FM2S (frequency moveto-set) for the reasons that we discuss shortly; the algorithm is defined by:

\section{FM2S (Frequency move-to-set)}

Init: $\mathrm{V}_{\mathbf{i}}=0$ for each item $\mathbf{i}$

At the t-th user selection with item i selected:

If item $\mathbf{i}$ not in the suggestion set $\mathrm{S} \mathrm{V}_{\mathbf{i}} \leftarrow \mathrm{V}_{\mathbf{i}}+1$

$\mathrm{E}=\{\mathbf{j} € \mathrm{~S}: \mathrm{V} \mathbf{J}<\mathrm{V} \mathbf{i}\}$

If $\mathrm{E}$ is nonempty

Remove a random item from $\mathrm{S}$ that is in $\mathrm{E}$

Add $\mathrm{i}$ to $\mathrm{S}$

Fig:4 FM2S Algorithm.

For every item,FM2S algorithm uses a counter variable for representing the number of users who have selected the particular items, which were not suggested in the item list. Furthermore, a selected item that was not suggested does not immediately qualify for entry in the suggestion set (as with M2S) but only if its counter exceeds the item that is already in the suggestion set.

In this section let us consider how the user select's items. User selects an item from the entire set of items by sampling, using the true item popularity distribution $r$. Where 


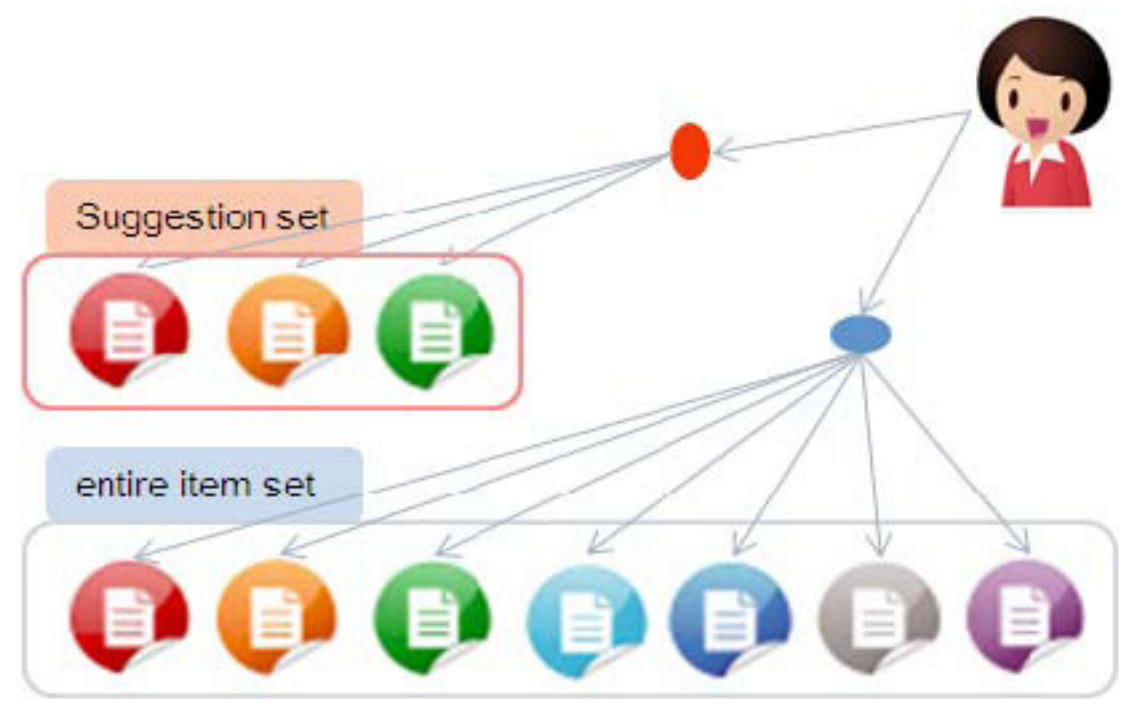

Fig 5: The Proposed Suggestion Set to the user

\section{Present Work}

\subsection{PROPOSED ALGORITHM}

In this paper we propose Naive Bayes algorithm for constructing a decision tree. We would like to give ranking for popular items based on a decision tree. If a learning algorithm produces accurate class probability estimates, it certainly produces an accurate ranking. But the opposite is not true. For example, assume that $E+$ and $E_{j}$ are a positive and a negative example respectively, and that the actual class probabilities are $p(+j E+)=0: 9$ and $p\left(+j E_{i}\right)=0: 4$. An

algorithm that gives class probability estimates: ${ }^{\wedge} p(+j E+)=0: 5$ and ${ }^{\wedge} p\left(+j E_{i}\right)=0: 45$, gives a correct order of $E+$ and $E_{j}$ in the ranking, although the probability estimates are poor. In the ranking problem, an algorithm tolerates the error of probability estimates to some extent, which is similar to that in classifcation.Recall that a classifcation algorithm gives the correct classifcation on an ex-ample, as long as the class with the maximum posterior probability estimate is identical to the actual class.Naive Bayes is easy to construct and has surprisingly good performance in classifcation, even though the conditional independence assumption is rarely true in real-world applications. On the other hand, naive Bayes is found to produce poor probability estimates .

TRAINMULTINOMIALNB(C,D)

$1 \mathrm{~V} \leftarrow$ ExtractVocabulary(D)

$2 \mathrm{~N} \leftarrow \operatorname{CountDocs}(\mathrm{D})$

3 for each cEC

4 do Nc $\leftarrow$ CountDocsInClass(D,c)

5 prior $[\mathrm{c}] \leftarrow \mathrm{Nc} / \mathrm{N}$

6 text $_{\mathrm{c}} \leftarrow$ ConcatenateTextOfAllDocsInClass(D,c)

7 for each $t \in V$

8 do Td $\leftarrow$ COUNTTOKENOFTERM(textc,t) 
International Journal of Computer Science \& Information Technology (IJCSIT) Vol 4, No 1, Feb 2012

9 for each $\mathrm{t} \in \mathrm{V}$

10 do condprob $[\mathrm{t}][\mathrm{c}] \leftarrow \mathrm{Tct}+1 / \sum \mathrm{dt} \mathrm{Tdt}+1$

11 return $\mathrm{V}$,prior,condprob

Fig 6:Ranking Popular Items using TRAINMULTINOMIALNB

ApplyMultinomialNB(C,V,prior,condprob,d)

1 for each $W \quad \operatorname{ExtractTokensFromDoc}(V, d)$

2 for each $c \in \mathbf{C}$

3 do score $[\mathrm{c}] \leftarrow \log$ prior $[\mathrm{c}]$

4 for each $t \in W$

5 do score $[\mathrm{c}]+=\log$ condprob $[\mathrm{t}][\mathrm{c}]$

6 return arg max score[ c]

Fig: 7 Ranking Popular Items using ApplyMultinomialNB ( Code for Ranking Popular Items using Naïve Bayes theory )

$\mathrm{C}$ is a fixed set of classes and it is given as $\mathrm{C}=\{\mathrm{c} 1, \mathrm{c} 2, \ldots, \mathrm{cJ}\}$. A training set $\mathrm{D}$ of labeled documents with each labeled Document $(\mathrm{d}, \mathrm{c}) € \mathrm{X} \times \mathrm{C}$ Using a learning method or learning algorithm, we then wish to learn a classifier that maps documents to classes: $: \mathrm{X} \rightarrow \mathrm{C}$

\section{DESIGN \& IMPLEMENTATION}

In this paper the proposed and studied Naïve Bayes algorithm was used to design a framework for ranking and suggesting popular items for the users. Initially to add item sets into the framework we used the pseudo code in Fig.9 .And when the user wants to view the item sets a pseudo code is proposed in Fig. 10. And when we want to update the popular item sets a pseudo code is proposed in Fig. 6.And when the item sets those are not utilized can be deleted using the pseudo code is proposed in Fig. 7.

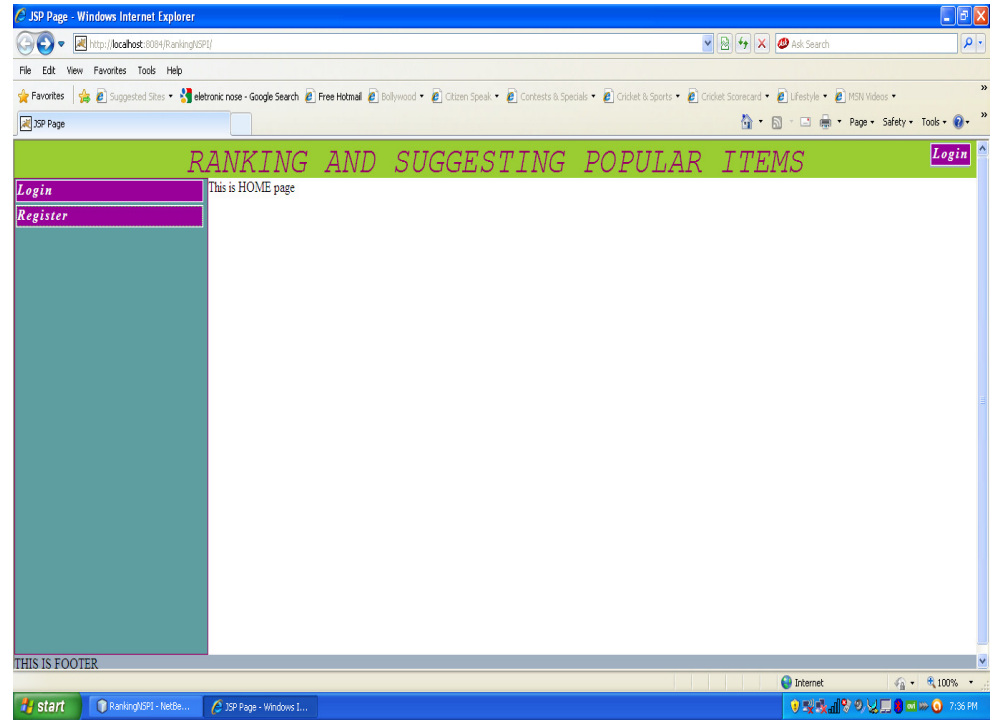

Fig: 8 Home Page 
International Journal of Computer Science \& Information Technology (IJCSIT) Vol 4, No 1, Feb 2012

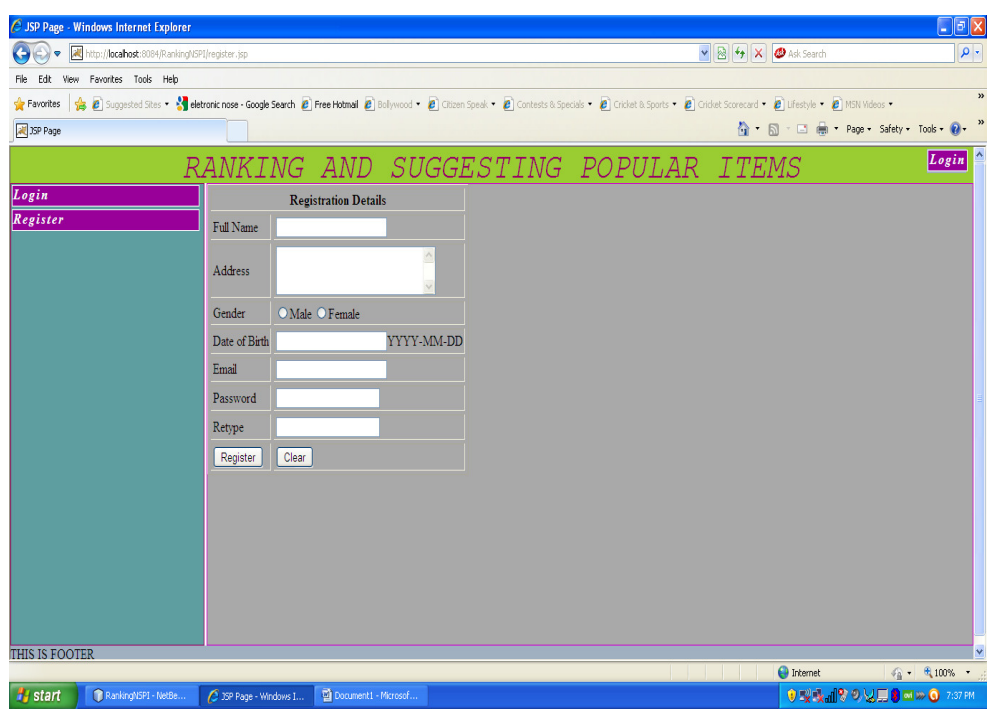

Fig 9 : Registration Form.

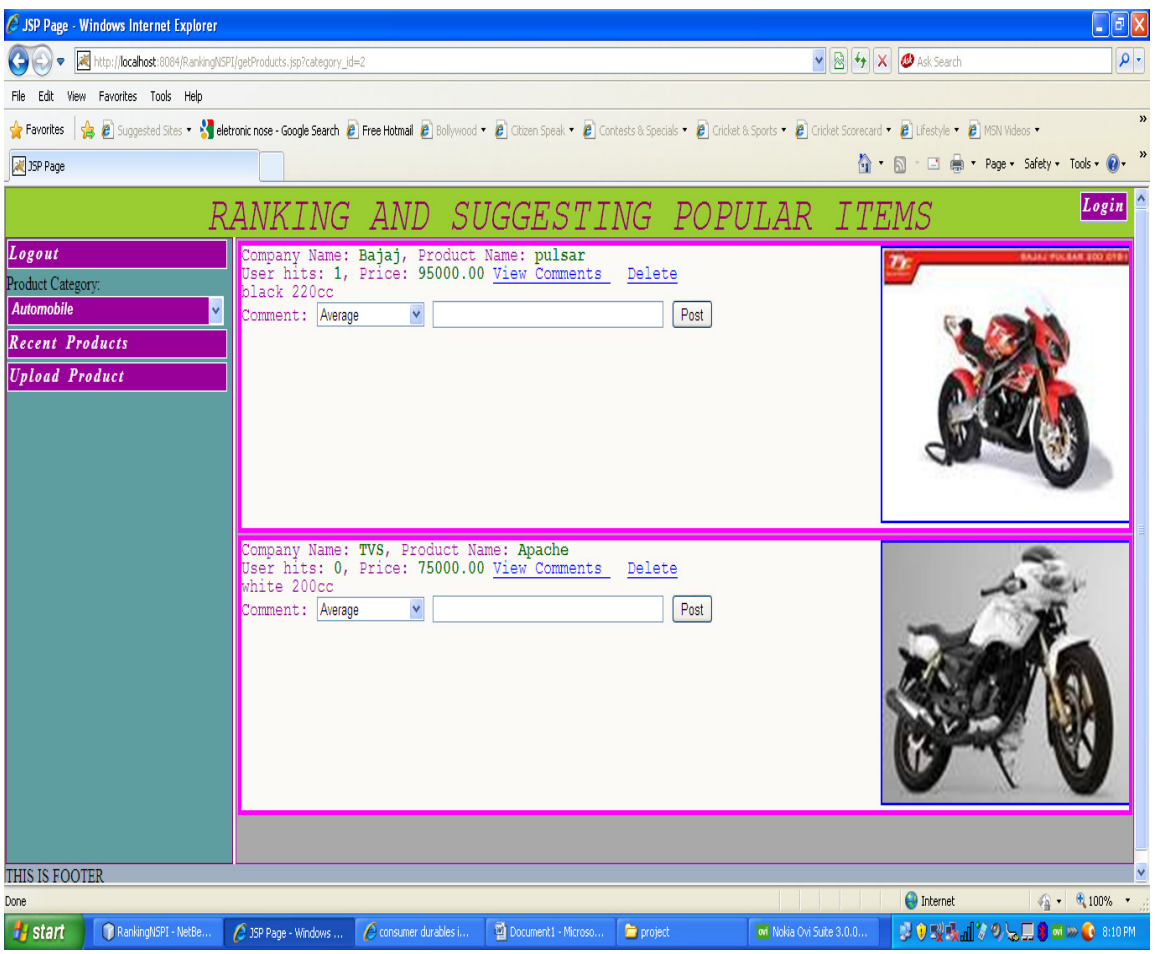

Fig 10 : Login Page 
International Journal of Computer Science \& Information Technology (IJCSIT) Vol 4, No 1, Feb 2012

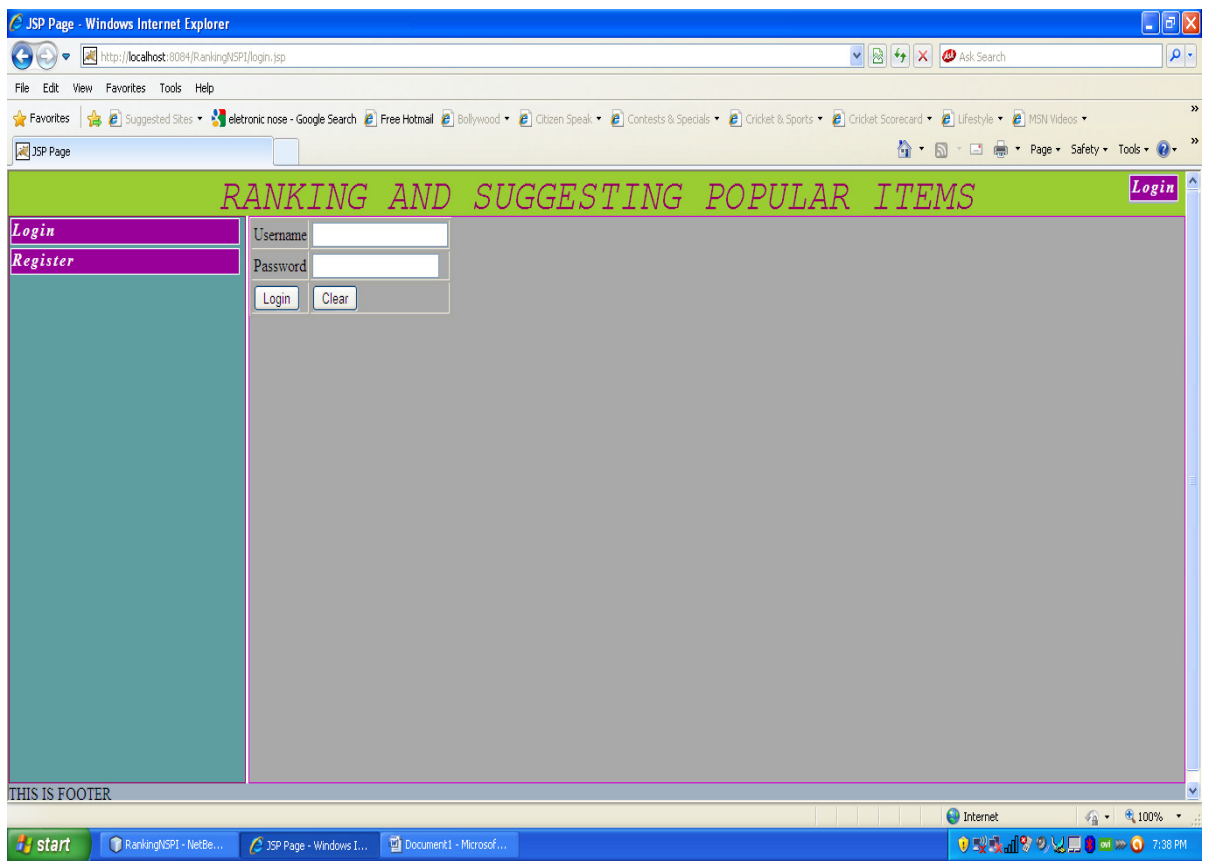

Fig 11: Items in the Iemset

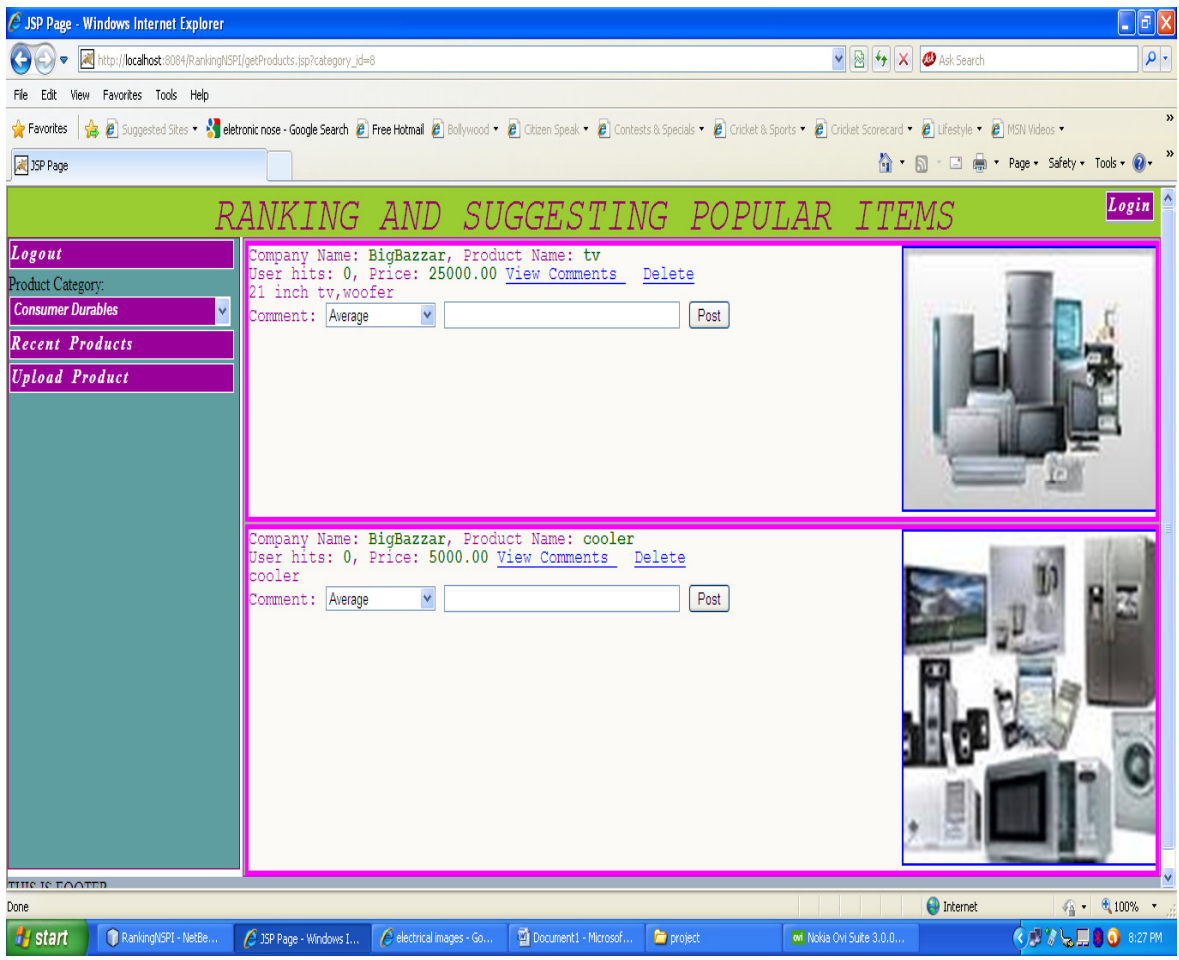

Fig 12: Items in the Itemset. 
International Journal of Computer Science \& Information Technology (IJCSIT) Vol 4, No 1, Feb 2012

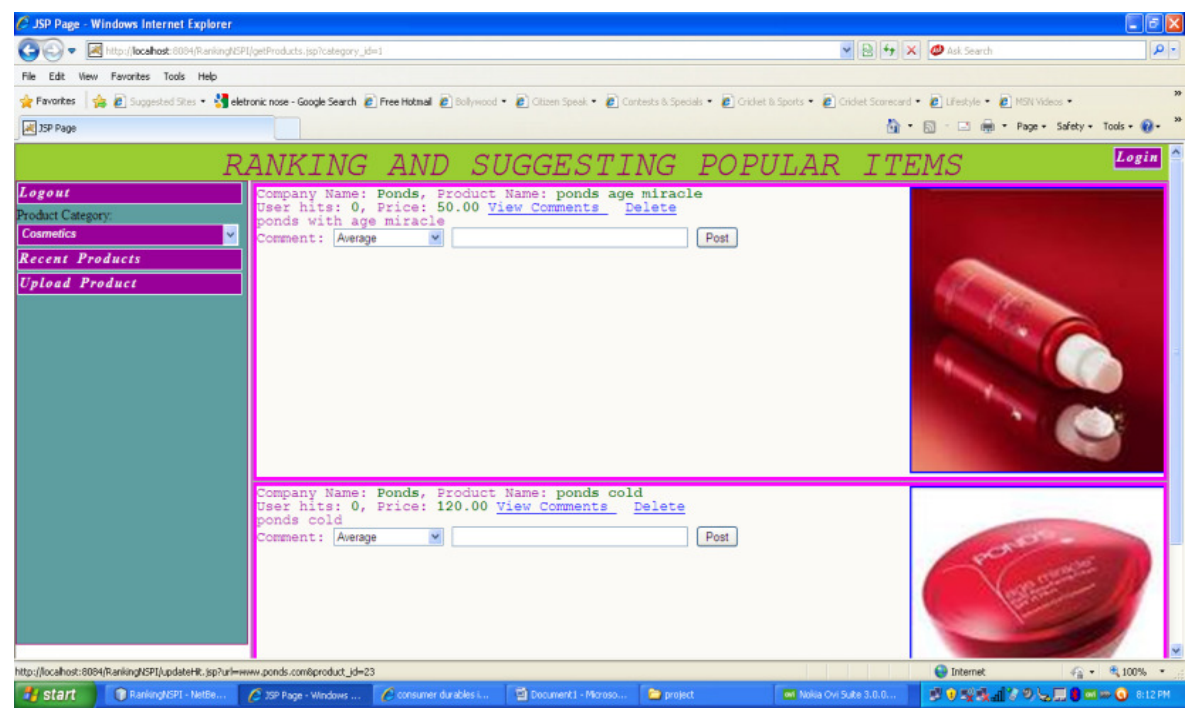

Fig 13 : Items in the Itemset.

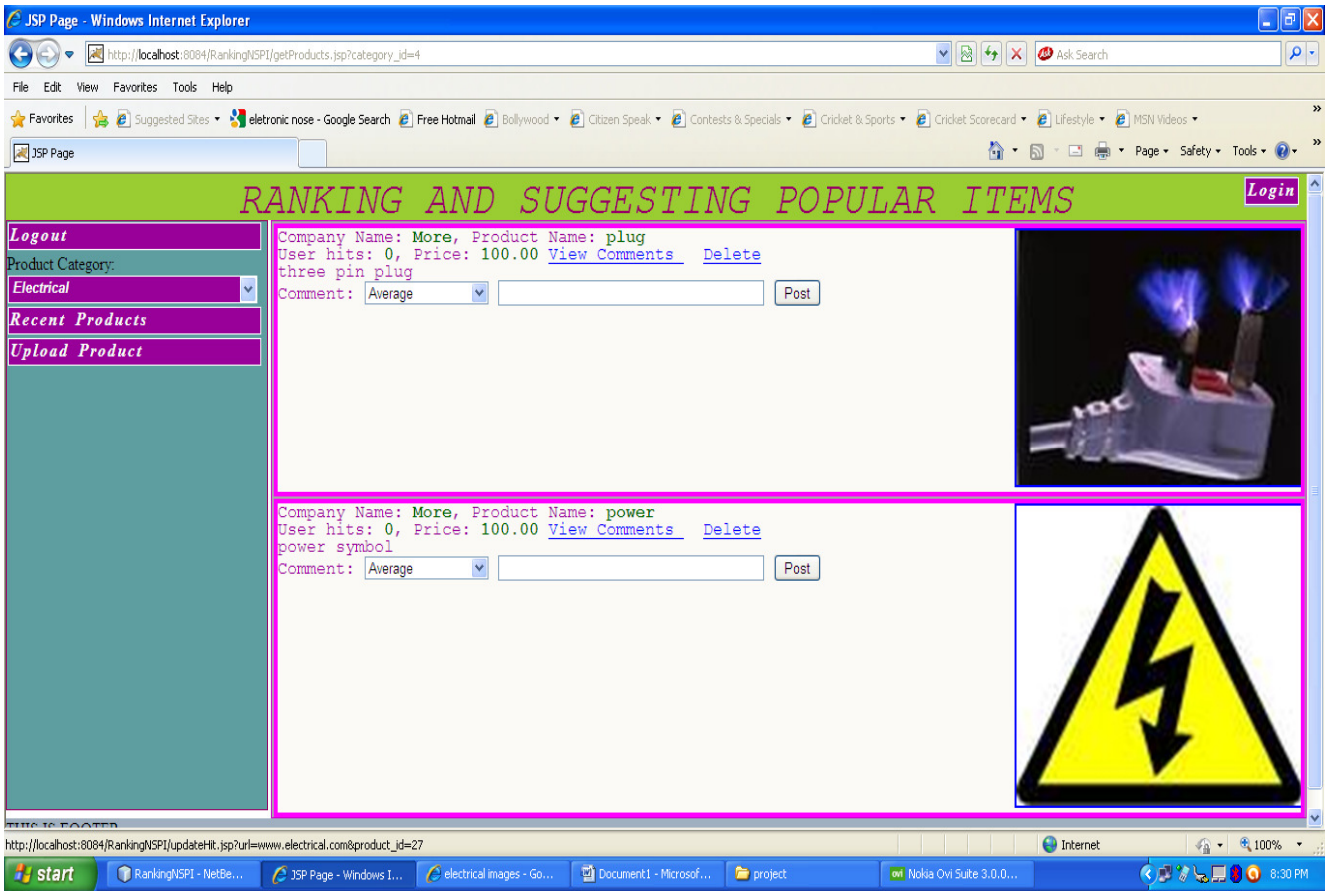

Fig 14: Items in the Itemset. 


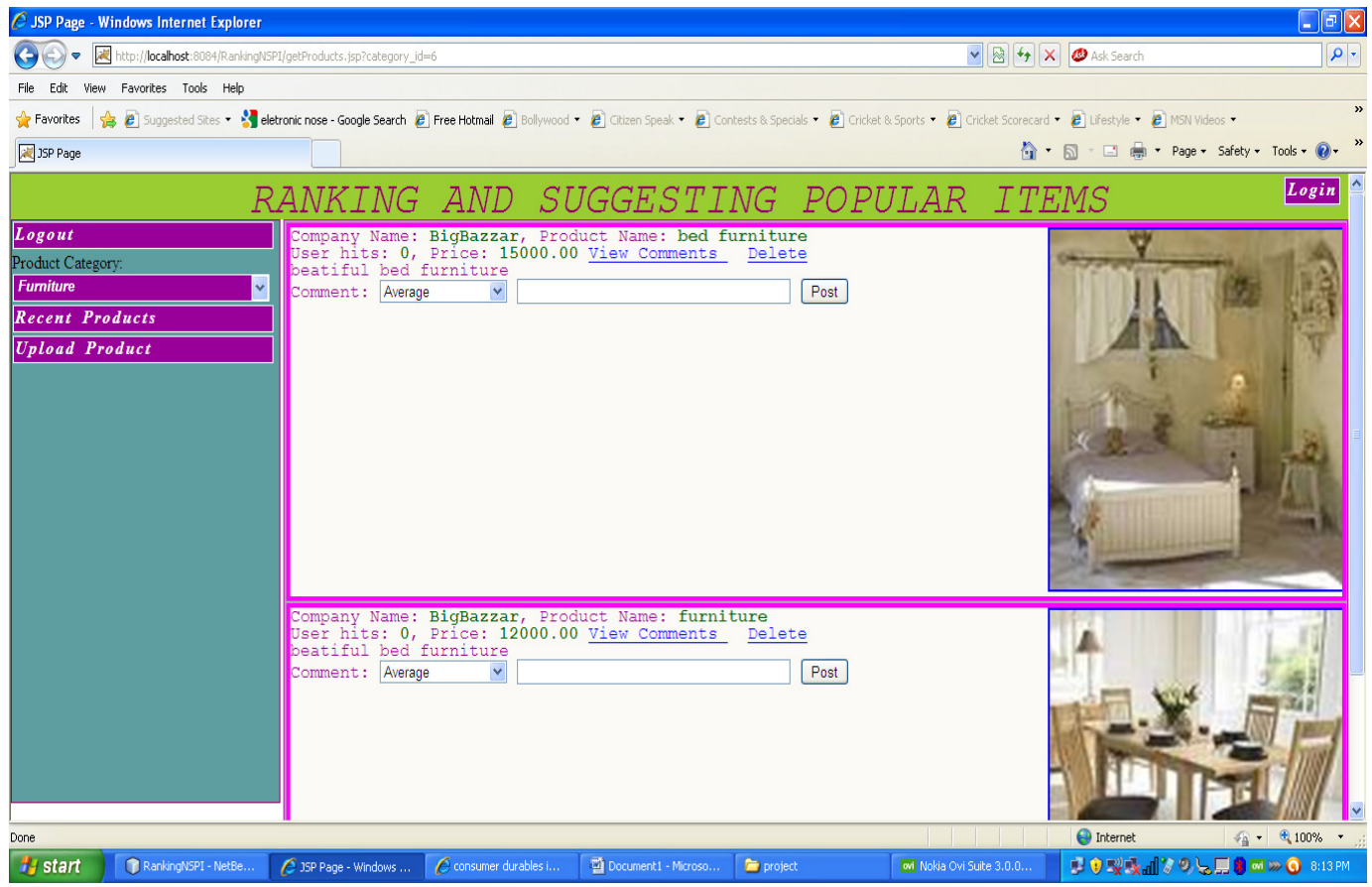

Fig 15: Items in the Iemset.

\section{CONCLUSION}

In this paper we analyzed randomized algorithms like naive, PROP, M2S, FM2S and proposed Naïve Bayes algorithm to suggest the popular items based on ranking and popularity of items. We have considered the problem of ranking and suggesting popular items in systems where users can select any subset of items and the users' selection of items can be swayed to the suggested items. In this paper we are proposing Naïve Bayes algorithm for ranking popular items based on probabilistic theory using Bayes theory concepts. The problem studied in this paper appears to differ from previous studies and may be worth exploring further We identified the limit ranking of the items which are provided by the algorithms and how they are related to the true popularity ranking and assessed the quality of suggestions as measured by the true popularity of suggested items.

\section{Acknowledgments}

We extremely thank our Principal and the management for their continuous support in Research and Development. We are also very grateful to our faculty members for their valuable suggestions and their ever ending support. Especially, we thank our college Principal and management for their financial support for receiving the sponsorship. 
International Journal of Computer Science \& Information Technology (IJCSIT) Vol 4, No 1, Feb 2012

\section{References}

[1] S. Brams and P. Fishburn, Approval Voting. Birkhauser, 1983.

[2] J. Cho, S. Roy, and R.E. Adams, "Page Quality:In Search of an Unbiased Web Ranking," Proc. ACM SIGMOD '05, 2005.

[3] S. Golder and B.A. Huberman, "The Structure of Collaborative Tagging Systems," J. Information Science, vol. 32, no. 2, pp. 198-208, 2006.

[4] J. Kleinberg and M. Sandler, "Using Mixture Models for Collaborative Filtering," Proc. 36th Ann. ACM Symp. Theory of Computing (STOC), 2004.

[5] R. Kumar, P. Rajagopalan, and A. Tomkins, "Recommendation Systems: A Probabilistic Analysis," Proc. 39th Ann. Symp. Foundations of Computer Science (FOCS), 1998.

[6]T.L.Lai and H.Robbins, “Asymptotically Efficient Adaptive Allocation Rules,”Advances in AppliedMath., vol. 6, pp. 4-25, 1985.

[7] R.M. Phatarfod, "On the Matrix Occurring in a Linear Search Problem," J. Applied Probability, vol. 18, pp. 336-346, 1991.

[8] G. Salton and M.J. McGill, Introduction to Modern Information Retrieval. McGraw-Hill Education, 1983.

[9] S. Sen, S.K. Lam, A.-M. Rashid, D. Cosley, D. Frankowski, J. Osterhouse, F.M. Harper, and J. Riedl, "Tagging, Communities, Vocabulary, Evolution," Proc. 2006 20th Anniversary Conf. Computer Supported CooperativeWork (CSCW), 2006.

[10] F. Suchanek, M. Vojnovi_c, and D. Gunawardena, "Social Tagging: Meaning and Suggestions," Proc. 17th ACM Conf. Information and Knowledge Management (CIKM), Oct. 2008.

[11] Z. Xu, Y. Fu, J. Mao, and D. Su,"Towards the Semantic Web: Collaborative Tag Suggestions," Proc. Workshop Collaborative Web Tagging Workshop at the WWW 2006, May 2006.

[12] V. Anantharam, P. Varaiya, and J. Walrand, "Asymptotically Efficient Allocation Rules for the Multiarmed Bandit Problem with Multiple Plays-Part i: i.i.d. Rewards," IEEE Trans. Automatic Control, vol. 32, no. 11, pp. 968-976, Nov. 1987.

[13] J.R. Anderson, “The Adaptive Nature of Human Categorization," Psychological Rev., vol. 98, no. 3, pp. 409-429, 1991.

[14] A.L. Baraba'si and R. Albert,"Emergence of Scaling in Random Networks," Science, vol. 286, pp. 509-512, 1999.

[15] S. Brams and P. Fishburn, Approval Voting. Birkhauser, 1983.

[16] S. Chakrabarti, A. Frieze, and J.Vera, "The Influence of Search Engines on Preferential Attachment," Proc. Symp. Discrete Algorithms (SODA), 2005.

[17] J. Cho, S. Roy, and R.E. Adams, "Page Quality: In Search of an Unbiased Web Ranking," Proc. ACM SIGMOD '05, 2005.

[18] Linden, G.; Smith, B.; York, J.; "Amazon.com recommendations: item-to-item collaborative filtering",Internet Computing, IEEE,Volume: 7 Issue:1-On page(s): 76 - 80

[19] Junqiang Liu,Yunhe Pan"Mining frequent item sets by opportunistic projection",ISBN:158113-567-X doi $>10.1145 / 775047.775081$

[20] Shilad Sen,Jesse Vig,John Riedl,"Tagommenders: connecting users to items through tags",WWW '09 Proceedings of the 18th international conference on World wide web. 
International Journal of Computer Science \& Information Technology (IJCSIT) Vol 4, No 1, Feb 2012

[21] S Goldwasser,S Micali,C Rackoff,"The knowledge complexity of interactive proofsystems",ISBN:0-89791-151-2 doi>10.1145/22145.22178

[22] Dwayne Bowman"Identifying the items most relevant to a current query based on items".

[23] T. M. Liggett. Interacting particle systems. Springer, 2 edition, 2006.

[24] S. Pandey, S. Roy, C. Olston, J. Cho, and S. Chakrabarti. Shuffling stacked deck: The case for partially randomized ranking of search engine results. In Proc. of VLDB '05, Trondheim, Norway, 2005.

[25] F. Radlinski and T. Joachims. Active exploration for learning rankings from clickthrough data. In Proc. of ACM KDD’07, San Jose, California, USA, 2007.

[26] M. Vojnovié, J. Cruise, D. Gunawardena, and P. Marbach. Ranking and suggesting tags in collaborative tagging applications. Technical Report MSR-TR-200706, Microsoft Research, February 2007.

[27] http://www.scribd.com/doc/47655582/Ranking-and-Suggesting-Popular-Items

[28] http://www.arnoldkling.com/apstats/bayes.html

[29] http://arnoldkling.com/apstats/chapter6.html

[30] http://www.slideshare.net/Tommy96/oracle-data-mining-concepts

[31] http://download.oracle.com/docs/cd/E11882_01/datamine.112/e16808/algo_nb.htm

First Author: ShiramShetty Gouthami is presently working as Asst.Prof in Computer Science Engineering Department at Jayamukhi Institute of Technological Sciences; Warangal for the past 5 years. She received her M.Tech Degree from JNTU Hyderabad,( A.P, India) in 2011.

Second Author: G.Jose Mary is presently working as Asst.Prof in Computer Science Engineering Department at Jayamukhi Institute of Technological Sciences; Warangal for the past 7 years. She received his M.Tech Degree in 2011.

Third Author: Pulluri Srinivas Rao is presently working as Head for the department of Information Technology at Jayamukhi Institute of Technological Sciences, Warangal.A.P (INDIA).Presently he is a research scholar at RU(A.P,India). His research areas include Data mining and Network Security. 